\title{
Radiation Testing Campaign for a New Miniaturised Space GPS Receiver
}

\author{
Craig Underwood \\ Surrey Space Centre \\ University of Surrey, Guildford, Surrey, UK \\ c.underwood@surrey.ac.uk \\ Reno Harboe Sorensen \\ Estec / European Space Agency, NL \\ Reno.Harboe.Sorensen@esa.int
}

\begin{abstract}
This paper describes the radiation susceptibility testing and analysis of a miniaturised space Global Positioning System (GPS) receiver for small satellite applications. Tests on commercial-off-the-shelf (COTS) parts included total ionising dose (TID), single-event effect (SEE) testing and receiver operational effects under heavy ion exposure.

Keywords - GPS, Space GPS receiver, chipset, small satellites, radiation tests
\end{abstract}

\section{INTRODUCTION}

An increasing number of small satellites are proposed for miscellaneous applications, many of which assume the use of Global Positioning System (GPS) for positioning, timing and orbit determination. The smallest satellites are only $1 \mathrm{~kg}$ in mass, which is smaller than most commercially available space GPS receivers. Equally demanding are the requirements for power consumption, where 1 watt is considered excessive, and also low cost, implying the use of commercial component based GPS receivers. Small commercial-based GPS receivers have previously been flown in space [1][3], and this has shown that terrestrial miniaturisation of GPS technology can be successfully applied to space applications with due caution taken of the environment [2]. This paper describes the characterisation and adaptation of new commercial GPS technology for space use, including radiation testing, and preparation for demonstration on a satellite.

\section{THE SGR-05 GPS RECEIVER}

The Zarlink Semiconductors GP4020 uses $0.35 \mu \mathrm{m}$ Digital CMOS with single polysilicon and is the successor to the widely used GEC Plessey / Mitel GP2000 GPS chipset. It uses similar correlator technology to the GP2021, but an ARM7TDMI microprocessor is integrated onto the same IC, and with the 3.3 volt operation, a much lower power consumption is achieved. The Australian company Sigtec produces a GPS application board (MG5001) that permits customers to develop and test their own software, and this has been adapted by Surrey as a starting point for a new space receiver. Three versions of GPS receivers have been designed:

\author{
Martin Unwin \\ Surrey Satellite Technology Ltd \\ University of Surrey, Guildford, Surrey, UK \\ m.unwin@sstl.co.uk
}

\begin{abstract}
Adam Frydland, Peter Jameson
QinetiQ, Farnborough, Hants, UK

ADFrydland@space.qinetiq.com
\end{abstract}

- the $S G R-05 U$ uses largely the same hardware as the Sigtec COTS board, but with some component swaps and space-compatible software. This is intended for the lowest budget missions.

- the SGR-05P has error-detection and correction (EDAC)-protected memory and additional interfacing circuitry to support missions with a wider range of requirements.

- the SGR-GEO is based on the SGR-05P, with an oven-controlled crystal oscillator (OCXO), some additional power supply and interface circuitry, and specialised operating software. This receiver is intended to be used at medium-altitude Earth orbit (MEO) and geostationary Earth orbit (GEO) (experiments have shown that GPS signals can be tracked in GEO).

The first flights of the SGR-05U and the SGR-GEO are planned for 2005 on separate satellites as experiments.

\section{RADIATION TEST CAMPAIGN}

In collaboration with QinetiQ Ltd and ESA and with some funding from the BNSC, Surrey embarked on a radiation test campaign in an attempt to characterise the susceptibility of the new chipset to radiation expected in an orbital environment, both in terms of Total Ionising Dose (TID) and single event effects (SEE). Samples of the MG5001 receiver were obtained from Sigtec. These were adapted for the tests, and the GP4020 IC replaced with "delidded" GP4020s (see Fig.1).

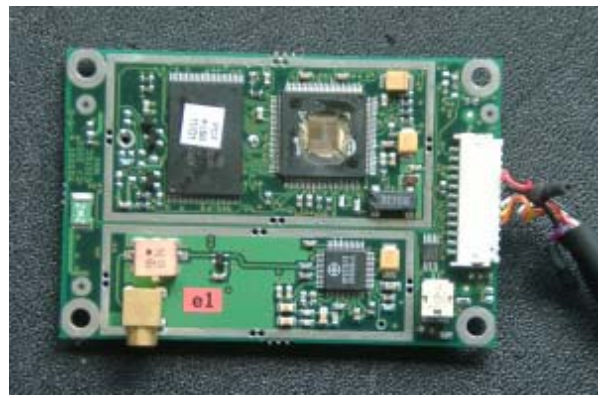

Figure 1. Test Application GPS Receiver Board Showing De-lidded GP4020 


\section{A. Total Ionising Dose Tests}

TID testing was performed at the University of Surrey in 2001 using a Cobolt-60 gamma radiation hot-spot source at a rate of $0.72 \mathrm{rad}\left(\mathrm{SiO}_{2}\right) \mathrm{s}^{-1}$ at room temperature The small size of the GPS receiver allowed the entire receiver to be radiated at once, enabling the characterisation of the whole receiver. The receiver was powered during the irradiation using a battery and a linear regulator circuit to ensure that the receiver was correctly biased and operating (although not attached to an antenna). The receiver was removed from the irradiation at regular intervals to test whether it was still operational or if had succumbed to the radiation and currents were recorded.

\section{B. Single Event Effect Testing}

In July 2002, the susceptibility of the GP4020 to SEEs was tested in a campaign at the UCL Belgian cyclotron Heavy Ion Facility. SEE testing was achieved by firing heavy ions at the device under test and observing susceptibility. The angle and species of the heavy ions can be selected to vary the effective linear-energy transfer (LET) of the ion to simulate the effects of typical radiation experienced in orbit due to cosmic rays. It is necessary to expose the silicon of the device directly to the ions, i.e. to de-lid the device. The etching process can sometimes damage the component, and so several devices are prepared in case of de-lidding failure. To enable proper test of the component, it must be embedded as part of an operational circuit so that radiation induced events can be monitored dynamically. Each device was therefore prepared and mounted in a separate GPS application circuit board.

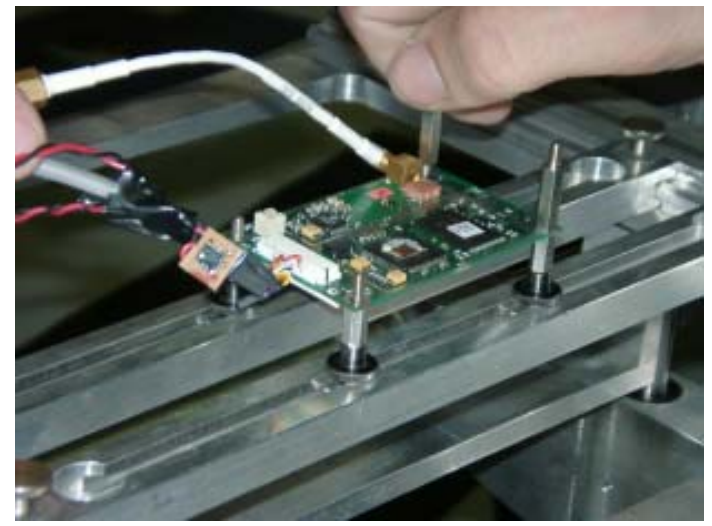

Figure 2. Device being Fitted to Tray for Heavy Ion Testing

An ARM assembly code program was written to operate on the GP4020 such that bit-patterns $\left(55_{h}, A_{h}, 00_{h}\right.$ and $\left.F_{h}\right)$ were written to the on-chip static random-access memory (SRAM) and registers to enable the detection bit flips due to heavy ions. The device was protected from single-event latch-ups (SELs) using a fast-acting current switch that could be monitored and reset by the PC. The results were logged using a PC program such that statistics of SELs and single-event upsets (SEUs) could be gathered, and micro-latch-ups ( $\mu \mathrm{LUs}$ ) could be observed using a current sensor linked to an analogue to digital converter card in the PC.

A final unusual test was made where the GPS receiver was operated in its full operational state, while the GP4020 was being subjected to heavy ion radiation. The test equipment permitted the connection to an antenna mounted externally to the building such that it was able to demonstrate tracking of GPS satellites and positioning.

\section{RESULTS}

\section{A. Total Ionising Dose Results}

The GPS receiver was exposed to radiation, and removed from the chamber about once every $3 \mathrm{kRad}\left(\mathrm{SiO}_{2}\right)$, to measure dynamic current and static current (holding the receiver in its reset state). Fig. 3 shows the static current starting to increase after about $10 \mathrm{kRad}\left(\mathrm{SiO}_{2}\right)$ of dose, and the receiver unit was able to tolerate between 11 and $15 \mathrm{kRad}\left(\mathrm{SiO}_{2}\right)$ of total dose before it failed. After annealing the receiver under bias at room temperature over night and then in a $100^{\circ} \mathrm{C}$ chamber for 10 hours, the receiver recovered some degree of functionality (although not enough to be usable). The cause of the failure was not identified, but the $11 \mathrm{kRad}\left(\mathrm{SiO}_{2}\right)$ system tolerance is adequate for many low cost space applications.

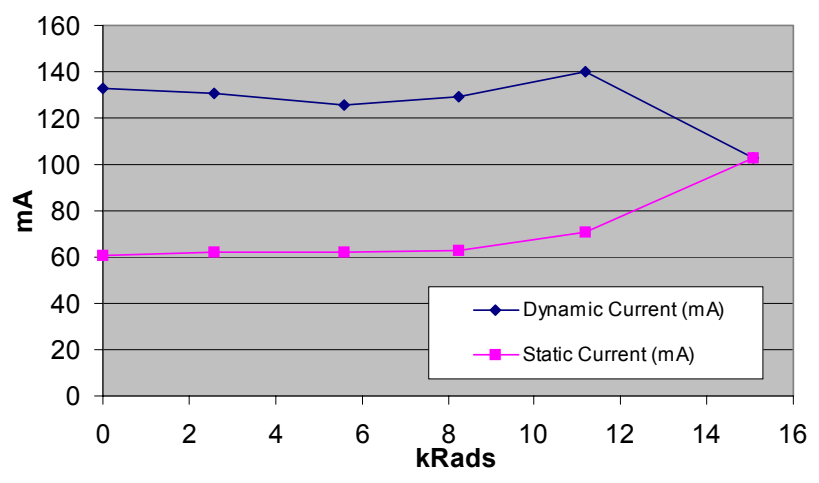

Figure 3. SGR-05U: Total Dose Effects on Nominal and Static Currents

\section{B. Single Event Effects Results}

The heavy ion testing proceeded successfully, and SRAM and register upsets, latch-ups, micro latch-ups, program crashes and resets were automatically counted using the PC software, and logged into result files. Latch-ups caused the protection switch to trip every time, and the devices under test apparently suffered no ill-effects. Micro-latch-ups were rare, but the current increases were captured using an analogue to digital card in the PC. An example of multiple micro-latch-ups is given in Figure 4.

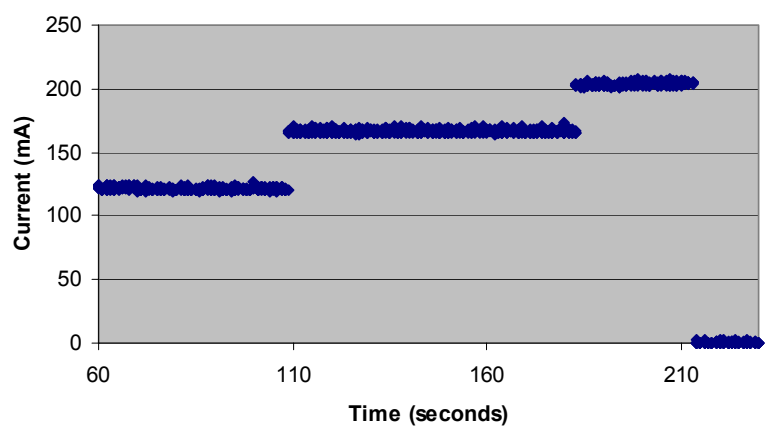

Figure 4. Micro-latch-up example: Argon, LET $=28.2 \mathrm{MeV} \mathrm{mg}^{-1} \mathrm{~cm}^{2}$ 
The results from the GP4020 heavy ion testing were collated from all the tests in order to gather the statistics for each type of SEE. LET thresholds and cross section characteristics were derived through fitting using Weibull distribution curves. For each set of results, the raw SEE-counts are turned into cross-sections $\left(\mathrm{cm}^{2} \mathrm{bit}^{-1}\right)$ by dividing the number of observed events by the fluence (ions $\mathrm{cm}^{-2}$ ) and by the target number of bits, or devices as appropriate. The data for each test LET are plotted with error-bars in cross-section calculated as the $95 \%$ confidence limits of an assumed Poisson process.

The curves fitted to the data are derived from a best-fit Weibull distribution. This distribution has the functional form of:

$\mathrm{F}(\mathrm{LET})=1-\exp \left\{-\left[\left(\mathrm{LET}-\mathrm{LET}_{\text {on-set }}\right) / \mathrm{W}\right]^{\mathrm{S}}\right\}$ for $\mathrm{LET}>\mathrm{LET}_{\text {on-set }}$ $\mathrm{F}(\mathrm{LET})=0$ for LET $<=\mathrm{LET}_{\text {on-set }}$ where $\mathrm{LET}_{\text {on-set, }}, \mathrm{W}$ and $\mathrm{S}$ are the fitting parameters.

The function is normalised by multiplying by the measured (or estimated) saturated cross-section $\left(\mathrm{cm}^{2} \mathrm{bit}^{-1}\right)$.

The radiation tests were self-consistent, and a good quantity and quality of measurements mean that the primary characteristics were well determined. Two samples were tested, and the results from both agreed closely.

The SRAM upset susceptibility is fairly high, as might be expected from commercial 3.3V technology, and the threshold

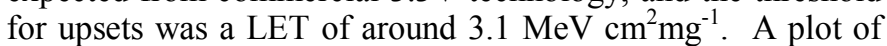
the results from the SRAM from one of the devices is shown in Fig. 5. It was found that bit flips from 1 to 0 and 0 to 1 were equally likely. A comparison of results from the GP4020 SRAM and GP4020 registers suggests that the registers may have a slightly higher saturated cross-section than the SRAM, although there are far fewer registers and consequentially fewer events (see Fig. 6).

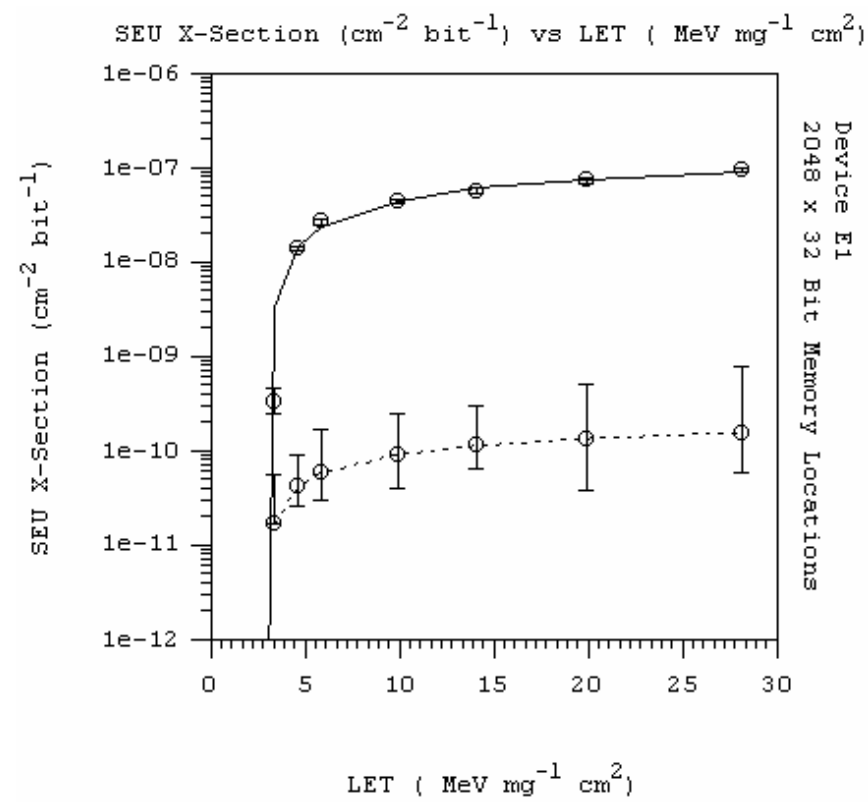

Figure 5. GP4020 SRAM SEU Cross-Section (Device “e1”), Dotted Line indicates MBU Cross-Section
Multiple-bit upset (MBU) statistics were also gathered: once again, the small number of events means poorly determined statistics, but it appears MBU cross section is approximately 500 times lower than that of the SRAM (Fig. 5). The MBUs were found to all be double bit upsets. Results between the two device samples ("e1" and "e3") were consistent in each case.

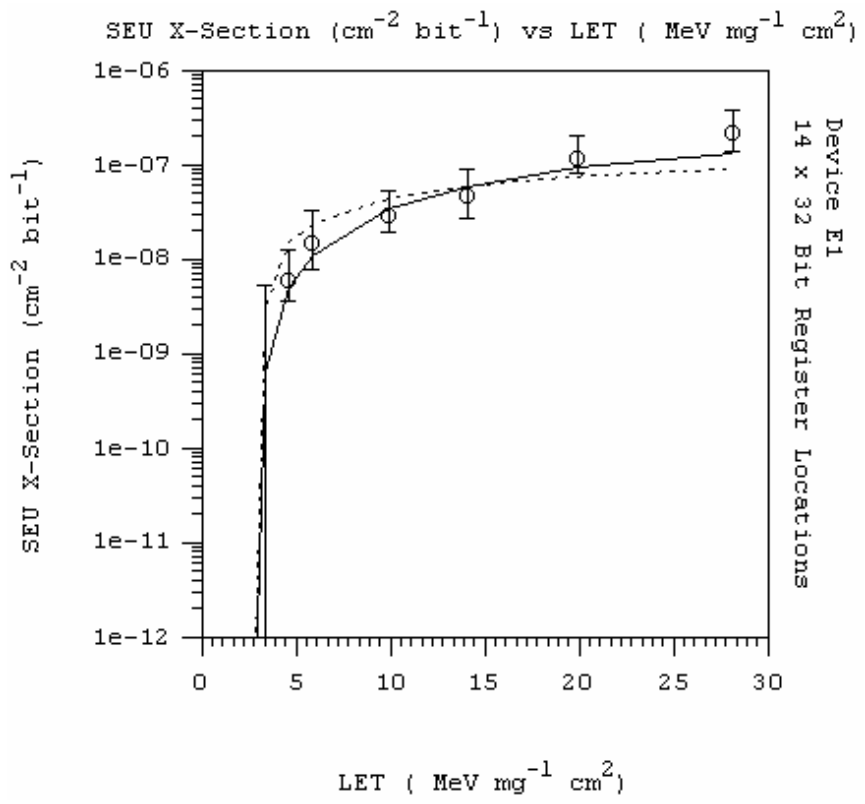

Figure 6. GP4020 Register SEU Cross-Section (Device "el") Dotted Line Indicates SRAM SEU Cross-Section

The GP4020 was significantly less susceptible to latch-ups than to SEUs and the SEL LET threshold lies between 14 to 20 $\mathrm{MeVcm}^{2} \mathrm{mg}^{-1}$ (see Fig. 7). Micro-latch-ups were also observed but only when testing at the highest LET $\left(28.2 \mathrm{MeV} \mathrm{cm}^{2} \mathrm{mg}^{-1}\right)$

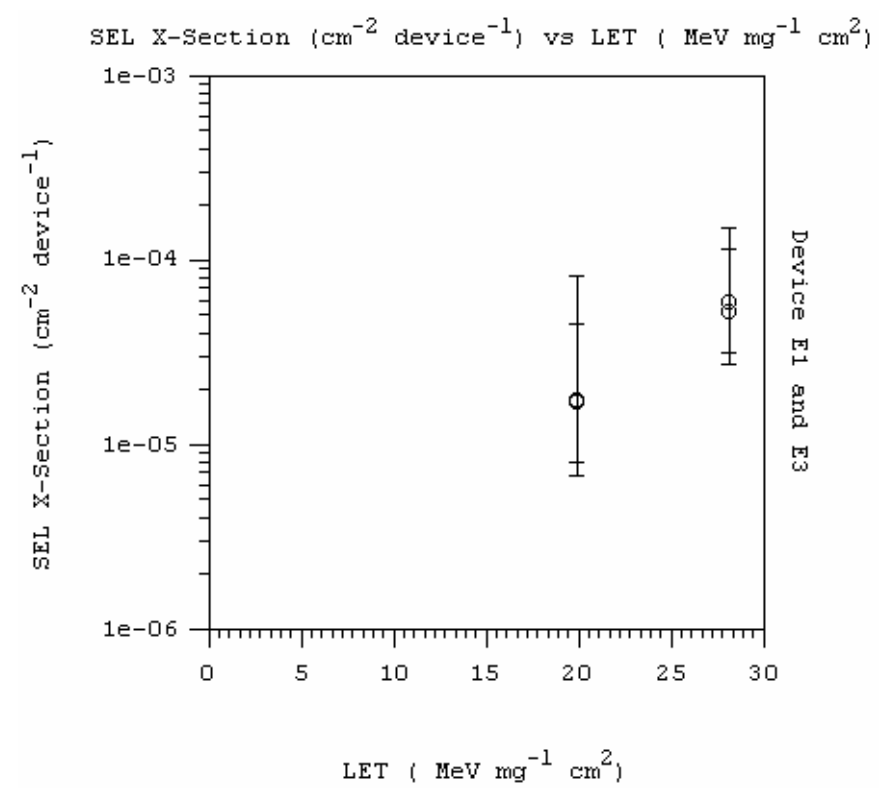

Figure 7. GP4020 SEL Cross-Section (Device "e1" and "e3") 
Other effects logged included Watchdog Timeouts and selfinduced Software Resets (see Figs 8, 9), together referred to as single-event functional interrupts (SEFIs). In common with the SEUs, the threshold is quite low. The data are consistent with a Weibull LET on-set of $3.2 \mathrm{MeV} \mathrm{cm} \mathrm{mg}^{-1}$.

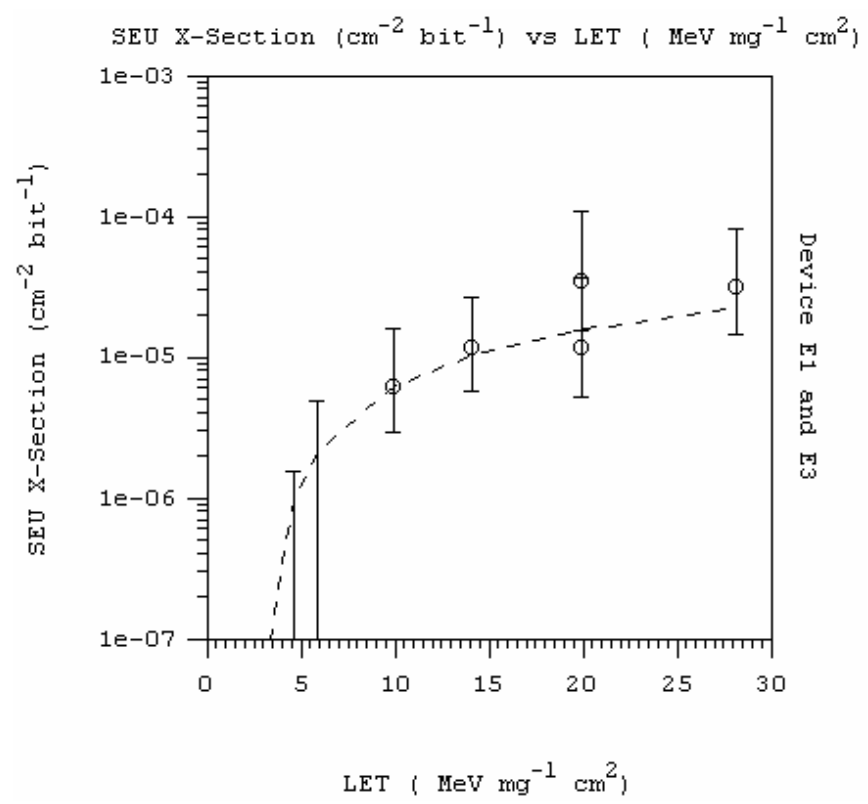

Figure 8. GP4020 Timeout Cross-Section (Device "e1")

Receiver Operational Effects: The receiver was tested while the GP4020 was radiated with low LET and low flux of heavy ions $\left(5.85 \mathrm{MeV} \mathrm{cm}^{2} \mathrm{mg}^{-1}\right.$ at 500 ions $\mathrm{cm}^{-2} \mathrm{~s}^{-1}$, and 14.1 $\mathrm{MeV} \mathrm{cm} \mathrm{mg}^{-1}$ at 400 ions $\mathrm{cm}^{-2} \mathrm{~s}^{-1}$ ). The receiver operation suffered significantly in this mode, and it failed to operate normally for more than a few tens of seconds (sustaining fluences of between 5000 and 14800 ions $\mathrm{cm}^{-2}$ ). This was an interesting test as the radiation flux under these circumstances is much higher than will be seen in orbit.

A list of the fitting parameters derived is given in Table I, and a summary of all the radiation test results is given in Table II.

\section{RADIATION MITIGATION IN DESIGN}

The results of the radiation tests can be compared to results from the 5V GPS chipset tested on a previous occasion [2]. The SRAM/register SEU saturated cross section of the GP4020 $\left(1.3-2.3 \times 10^{-7} \mathrm{~cm}^{2} \mathrm{bit}^{-1}\right)$ is similar to that of the ARM60B (2 $4 \times 10^{-7} \mathrm{~cm}^{2}$ bit $\left.^{-1}\right)$, but the threshold is lower $\left(3.1 \mathrm{MeV} \mathrm{mg}^{-1} \mathrm{~cm}^{2}\right.$ compared to $10 \mathrm{MeV} \mathrm{mg} \mathrm{cm}^{-1}$ ). This seems to confirm the general perception that, as commercial technologies migrate from $5 \mathrm{~V}$ to $3.3 \mathrm{~V}$, the sensitivity to SEEs increases.

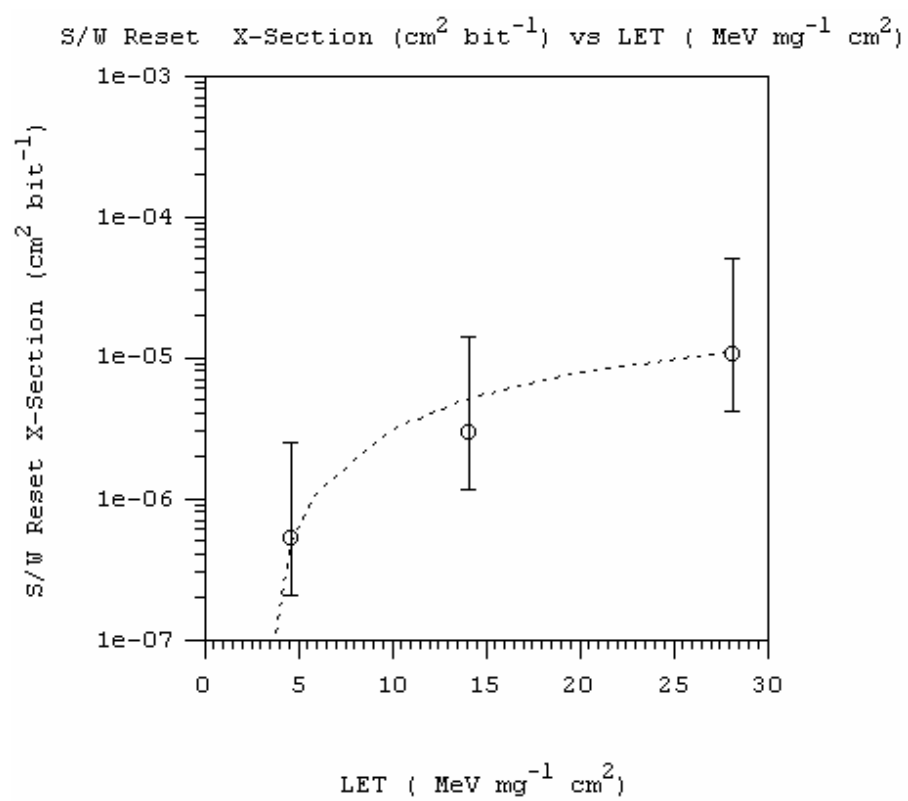

Figure 9. GP4020 Software Reset Cross-Section (Device "e1")

For low Earth orbit, the TID of the whole receiver is acceptable, as a lifetime of $\sim 10$ years is foreseen under average conditions, given $4 \mathrm{~mm}$ Aluminium screening. The receiver could also be used in higher orbits, e.g. GEO, given extensive shielding. If an equivalent of $10 \mathrm{~mm}$ of Aluminium is used or, for example, $3 \mathrm{~mm}$ of copper, it is calculated that the receiver will survive 7-10 years in GEO. The advantage of the SGR-05 is that its small size requires less shielding mass than most other electronic systems.

The SEU susceptibility is a matter of concern, as a significant number of upsets are to be expected in low Earth orbit. The operational tests give an idea how the receiver will behave when a large number upsets occur, with a failure to track signals consistently. The SGR-05U has no EDAC circuitry and will be sensitive to upsets, and so it is important for it to have a robust way of recovering. Fortunately, the GP4020 is equipped with a Watchdog timer, and this will generally permit the receiver to be up and positioning within 10

TABLE I. FitTED WEIBUll PARAMETERS

\begin{tabular}{|l|l|l|l|l|}
\hline SEE & $\begin{array}{l}\text { LET Onset } \\
\left(\mathrm{MeV} \mathrm{mg}^{-1} \mathrm{~cm}^{2}\right)\end{array}$ & $\begin{array}{l}\text { W } \\
\left(\mathrm{MeV} \mathrm{mg}^{-1} \mathrm{~cm}^{2}\right)\end{array}$ & S & Saturated Cross-Section \\
\hline SRAM SEU & 3.2 & 20.6 & 0.8 & $1.3 \times 10^{-1}\left(\mathrm{~cm}^{2} \mathrm{bit}^{-1}\right)$ \\
\hline MBU & 3.1 & 24.1 & 0.6 & $2.4 \times 10^{-10}\left(\mathrm{~cm}^{2} \mathrm{bit}^{-1}\right)$ \\
\hline Register SEU & 3.1 & 28.4 & 1.3 & $2.3 \times 10^{-1}\left(\mathrm{~cm}^{2} \mathrm{bit}^{-1}\right)$ \\
\hline Device Timeouts & 3.2 & 30 & 1.2 & $4 \times 10^{-9}\left(\mathrm{~cm}^{2} \operatorname{device}^{-1}\right)$ \\
\hline Device Software Resets & 3.2 & 30 & 1.2 & $2 \times 10^{-5}\left(\mathrm{~cm}^{2} \mathrm{device}^{-1}\right)$ \\
\hline
\end{tabular}


minutes after a Reset (or less if Almanac is retained in nonvolatile memory). Note that devices have been frequently flown by Surrey that have similar thresholds to the GP4020 without experiencing mission-limiting reliability problems (e.g. the 87C592 microcontroller).

To give greater confidence and reliability, the SGR-05P is being designed with external Triple Modular Redundancy (TMR) EDAC protected memory for speed and robustness, and software is likely to avoid the use of internal RAM on the GP4020. It is not possible to avoid using the registers but the chances of a register upset that causes a functional effect should be small enough to achieve an acceptable degree of robustness.

The expected latch-up rate is sufficiently low for a practical satellite sub-system. As with the earlier SGR-10/20 receivers a latch-up switch will be used that switches the receiver off rapidly after any current surge. SSTL's latch-up switch has been flown on several missions, and was also proven during the radiation test campaign. Micro-latch-ups are expected to be rare, but should ideally be checked for by the host computer. As with the SGR-10/20 design, a battery-backed non-volatile RAM is being avoided to ensure that all latch-ups can be caught and reset immediately. See also discussion in [4]. Other non-volatile memories should be used, for example Flash or FRAM memories. These cannot maintain a real-time clock, but will be able to maintain copies of GPS Almanac and user orbital elements during power cycling.

The test results indicate that SEFIs will occasionally happen. The GP4020 watchdog will usually help the receiver recover any effects from SEFIs, but there may be some SEFIs that cannot be solved through a self-induced reset. In this case, the host computer system will be required to monitor the receiver and cycle the power if anomalies are observed (e.g. lack of communications, or lack of position fix for an excessive period of time).

\section{CONCLUSIONS}

This paper has described the results and implications from a radiation test campaign aimed at assessing the susceptibility characteristics of the GP4020, a COTS GPS integrated circuit, for space applications. Three types of miniaturised GPS receivers have been developed based on the GP4020, and it is planned that two receivers will be demonstrated in orbit on flight opportunities in 2005. A future version of this receiver is also being designed that can use multiple front-ends to determine accurate attitude of a space vehicle. The radiation tests on the GP4020 suggest that with due care and attention, modern low power commercial GPS technology can be used in space applications, to be confirmed by a demonstration in orbit.

\section{ACKNOWLEDGMENT}

Thanks are due to QinetiQ, ESA for supporting the radiation tests and to BNSC for contributing towards the funding. SSTL also provided R\&D funding to support this work, and particular thanks go to David Helland and Guy Berger who helped in the test execution.

\section{REFERENCES}

[1] Unwin M J, Palmer P.L., Hashida Y, Underwood C.I., "The SNAP-1 and Tsinghua-1 GPS Formation Flying Experiment", Proc ION GPS 2000, Salt Lake City, Sept 2000

[2] Oldfield M.K. Underwood C.I, Unwin M.J., Harboe Sorensen R, Asenek V, "Pre-and Post-Flight Radiation Performance Evaluation of the Space GPS Receiver (SGR)", RADECS 1999, Sept 1999, Fontevraud, France, pp 418-423.

[3] Montenbruck O., Leung S, Bruninga R, "GPS Operations on the PCSat Microsatellite", Proc ION GPS 2002, Portland, Oregon, September 2002.

[4] Pritchard B, Swift G.M., Johnston A.H, "Radiation Effects Predicted, Observed and Compared for Spacecraft Systems", Radiation Effects Data Workshop, NSREC 2002.

TABLE II. GP4020 RADIATION TEST RESUlts SUMmary

\begin{tabular}{|c|c|c|c|c|}
\hline SEE & $\begin{array}{l}\text { GP4020 } \\
\text { SRAM }\end{array}$ & $\begin{array}{l}\text { GP4020 } \\
\text { Registers }\end{array}$ & $\begin{array}{l}\text { GP4020 } \\
\text { Device }\end{array}$ & $\begin{array}{l}\text { SGR-05U } \\
\text { Receiver }\end{array}$ \\
\hline SEU Threshold LET $\left(\mathrm{MeV} \mathrm{mg}^{-1} \mathrm{~cm}^{2}\right)$ & 3.2 & 3.1 & & \\
\hline Saturated SEU Cross Section $\left(\mathrm{cm}^{2}\right.$ bit $\left.^{-1}\right)$ & $1.3 \times 10^{-7}$ & $2.3 \times 10^{-7}$ & & \\
\hline MBU Threshold LET $\left(\mathrm{MeV} \mathrm{mg}^{-1} \mathrm{~cm}^{2}\right)$ & 3.1 & - & & \\
\hline Saturated MBU Cross Section $\left(\mathrm{cm}^{2} \mathrm{bit}^{-1}\right)$ & $2.4 \times 10^{-10}$ & - & & \\
\hline SEL Threshold LET $\left(\mathrm{MeV} \mathrm{mg}^{-1} \mathrm{~cm}^{2}\right)$ & & & $14-20$ & \\
\hline Saturated SEL Cross Section $\left(\mathrm{cm}^{2}\right.$ device $\left.{ }^{-1}\right)$ & & & $\sim 6 \times 10^{-5}$ & \\
\hline$\mu$ LU Threshold LET $\left(\mathrm{MeV} \mathrm{mg}^{-1} \mathrm{~cm}^{2}\right)$ & & & $20-28$ & \\
\hline Saturated $\mu$ LU Cross Section $\left(\mathrm{cm}^{2}\right.$ device $\left.{ }^{-1}\right)$ & & & $\sim 6 \times 10^{-5}$ & \\
\hline SEFI Threshold LET $\left(\mathrm{MeV} \mathrm{mg}^{-1} \mathrm{~cm}^{2}\right)$ & & & 3.2 & \\
\hline Saturated SEFI Cross Section $\left(\mathrm{cm}^{2}\right.$ device $\left.{ }^{-1}\right)$ & & & $\sim 2-4 \times 10^{-5}$ & \\
\hline \multicolumn{5}{|l|}{ TID } \\
\hline Total Dose to Failure $\mathrm{kRad}\left(\mathrm{SiO}_{2}\right)$ & & & & $11-15$ \\
\hline Lifetime in LEO with $4 \mathrm{~mm}$ Aluminium shielding & & & & $>10$ years \\
\hline Lifetime in GEO with $10 \mathrm{~mm}$ Aluminium shielding & & & & $>7$ years \\
\hline
\end{tabular}

